\title{
Preparation of a radiology department in an Italian hospital dedicated to COVID-19 patients
}

\author{
Anna Maria lerardi ${ }^{1}$ (1) Bradford J. Wood ${ }^{2} \cdot$ Antonio Arrichiello $^{1} \cdot$ Nicola Bottino $^{3} \cdot$ Laura Bracchi $^{4} \cdot$ Laura Forzenigo $^{1}$. \\ Maria Carmela Andrisani ${ }^{1}$. Valentina Vespro ${ }^{1}$. Cristian Bonelli ${ }^{5}$. Amel Amalou ${ }^{2}$. Evrim B. Turkbey ${ }^{6}$. \\ Baris I. Turkbey ${ }^{7}$. Giuseppe Granata ${ }^{1}$ - Antonio Pinto ${ }^{8}$. Giacomo Grasselli ${ }^{3,10}$. Nino Stocchetti ${ }^{9,10}$. \\ Gianpaolo Carrafiello ${ }^{1,11}$
}

Received: 23 June 2020 / Accepted: 2 July 2020 / Published online: 11 July 2020

(C) Italian Society of Medical Radiology 2020

\begin{abstract}
Preparedness for the ongoing coronavirus disease 2019 (COVID-19) and its spread in Italy called for setting up of adequately equipped and dedicated health facilities to manage sick patients while protecting healthcare workers, uninfected patients, and the community. In our country, in a short time span, the demand for critical care beds exceeded supply. A new sequestered hospital completely dedicated to intensive care (IC) for isolated COVID-19 patients needed to be designed, constructed, and deployed. Along with this new initiative, the new concept of "Pandemic Radiology Unit" was implemented as a practical solution to the emerging crisis, born out of a critical and urgent acute need. The present article describes logistics, planning, and practical design issues for such a pandemic radiology and critical care unit (e.g., space, infection control, safety of healthcare workers, etc.) adopted in the IC Hospital Unit for the care and management of COVID-19 patients.
\end{abstract}

Keywords COVID-19 $\cdot$ SARS-CoV-2 $\cdot$ Radiology $\cdot$ Pandemic $\cdot$ Hospital

\section{Introduction}

The Coronavirus Disease 2019 (COVID-19) pandemic began in Late 2019 in Wuhan, China. The outbreak is due to severe acute respiratory syndrome coronavirus 2 (SARS$\mathrm{CoV}-2$ ) infection [1]. Italy had precious little time to prepare any response to the COVID-19 pandemic. This response

Anna Maria Ierardi

amierardi@yahoo.it

1 Radiology Department, Fondazione IRCCS Cà Granda Ospedale Maggiore Policlinico, Via Francesco Sforza, 35, 20122 Milan, Italy

2 Center for Interventional Oncology, Radiology and Imaging Sciences, NIH Clinical Center and National Cancer Institute, Center for Cancer Research, National Institutes of Health, Bethesda, MD, USA

3 Department of Anesthesia and Critical Care, Fondazione IRCCS Cà Granda Ospedale Maggiore, Milan, Italy

4 Cerba Healthcare Italia, sede CURIE, Viale Liguria, 37, 20093 Cologno Monzese, MI, Italy

5 Healthcare Professionals Department, Foundation IRCCS Cà Granda Ospedale Maggiore Policlinico, Milan, Italy is led by the Italian national government in coordination and synchrony with local (or regional) governments and hospitals.

The major hot spot of COVID-19 patients have thus far been in the north of Italy, especially in the region of Lombardy. To date, the total of infections in Italy is $>215,000$, of which 80,723 are in Lombardy [2]. Over 10 million people

6 Radiology and Imaging Sciences, National Institutes of Health, Bethesda, MD, USA

7 Molecular Imaging Branch, National Cancer Institute, National Institutes of Health, Bethesda, MD, USA

8 Department of Radiology, CTO Hospital, Azienda dei Colli, Naples, Italy

9 Neuroscience Intensive Care Unit, Department of Anesthesia and Critical Care, Fondazione IRCCS Cà Granda Ospedale Maggiore Policlinico, Milan, Italy

10 Department of Pathophysiology and Transplantation, University of Milan, Milan, Italy

11 Department of Health Sciences, Università degli Studi di Milano, Milan, Italy 
live in Lombardy region, forming more than one-sixth of Italy's population, [3].

Within Lombardy, Milano's metropolitan area is the largest in Italy, and the third most populated functional urban area in the entire European Union [2].

As it is now well known from the clinical course of COVID-19 available in the guidelines and the literature, the most severe cases develop acute respiratory conditions that require intensive care unit (ICU) admission. Although only a few of those infected require ICU, the need for ICU beds increases exponentially during the upward trajectory of the pandemic outbreak curve. In this scenario, the demand for critical care in the northern region of Italy exceeded supply, raising significant practical and ethical concerns in the process. To overcome this important issue, within 2 weeks from concept to opening, a new hospital was arranged. This is not a camp hospital or acute triage "fever clinic," but a real "brick and mortar" hospital focused on intensive care and intensive triage of the critically ill cases. Two pavilions, located on two floors, of an exhibition area were made eligible to host all the equipment and staged care triage settings of the IC ward. All the services (laboratories, radiology, etc.) necessary to an independent functioning of over dedicated IC 200 beds were allocated in the same building with a strategic position. This location minimized crosscontamination and interactions with non-COVID-19 activities and staff. Procedural and staff sequestration that mimics the geographic sequestration of such an effort is requisite to successful nosocomial containment of pandemic COVID-19. Radiology is one of the frontline specialities in the clinical service, because of its role in diagnosis and follow-up of patients. A multidisciplinary symphony of coordinated policies, infrastructure, communications, flow, and staff awareness were harmonized to enable a successful mission to fight COVID-19. The present article describes the logistical setting, strategy, and organization for radiology services in a new dedicated Covid-19 critical care and critical triage hospital.

\section{Hospital}

A well-equipped dedicated hospital facility to deal with COVID-19 patients with adequate protective equipment and policies for healthcare workers is the key to successful delivery of safe and optimal public health care in the setting of a pandemic. The hospital was designed to be practically a self-contained and self-sufficient establishment that can meet most of its daily needs with only essential and limited contact with the outside world, via predetermined limited channels for such critical pipelines as nutritional services and other supply chains. Basic requirements that needed to be defined included: appropriate cleaning practices; adequate floor space for beds; adequate (ideally negative) one-pass ventilation for isolation rooms and procedure rooms; adequate isolation facilities for airborne, droplet, contact isolation and protective environment with well-defined and disseminated standard operating procedures (SOPs); and finally a regulated and rational traffic flow to minimize exposure or cross-contamination of high-risk patients and facilitate patient and clinical material transport.

The hospital, composed of 205 ICU beds includes 2 floors, is divided in red and green units or zones (Fig. 1). Red zones are completely dedicated to host beds for COVID-19 patients, ventilators and devices dedicated to IC management. Beds are contained in modules (from $\mathrm{A}$ to $\mathrm{H}$ as shown in the map (Fig. 2); in each module, 7 or 14 beds are located, depending on floor), with controlled access and unified connections to each other. Barrier nursing practices and protective isolation facilities are present to minimize risk of nosocomial infections. Beds are sequestered from each other as much as possible, to avoid cross-contamination, in case of different strains of COVID-19 or differing superinfections with more standard microorganisms. Part of the radiological services is also located in red zones (like CT, US, etc), laundry, access for patients, and patient triage is all located in the red zone also. Green zones include access for healthcare workers, changing rooms, offices and administrative area, and some support services. Such a staged space designation is analogous to the ante-room concept for staged doffing PPE, whereby the doffing occurs in staged locations, in very specific sequences, from very dirty to less dirty to more likely clean, in order to minimize human error and exposures. Doffing is done with a "Doffing Watcher" whose sole assignment is to watch the "doffers and donners" for human breaches in technique or sequence. Likewise it is wise to designate staff as "coordinators" for traffic control and communications of transport issues, and "educators" for dissemination of SOPs and new policies and practicing, and "runners" whose sole responsibility is to transfer equipment or supplies from a "clean" supply or storage space to a "dirty" procedure room, along predetermined and pre-practiced chains of transfer and chains of communication.

Green zones, as illustrated in the map (Fig. 1), are represented by an external perimeter, surrounding the red zones. Four entry points are present, equipped with protective personal equipment (PPE). Each red module is equipped with doffing points, from which it s possible to go out from the red zone. Attention to donning and doffing education, practicing, training, and standard SOPs is critical to safe operation of such a COVID-19 facility.

\section{Radiology service}

Radiology Unit is present in both red and green zones. In the red zones are located ultrasound (US) machines, one for each module; portable radiography is repeatable 


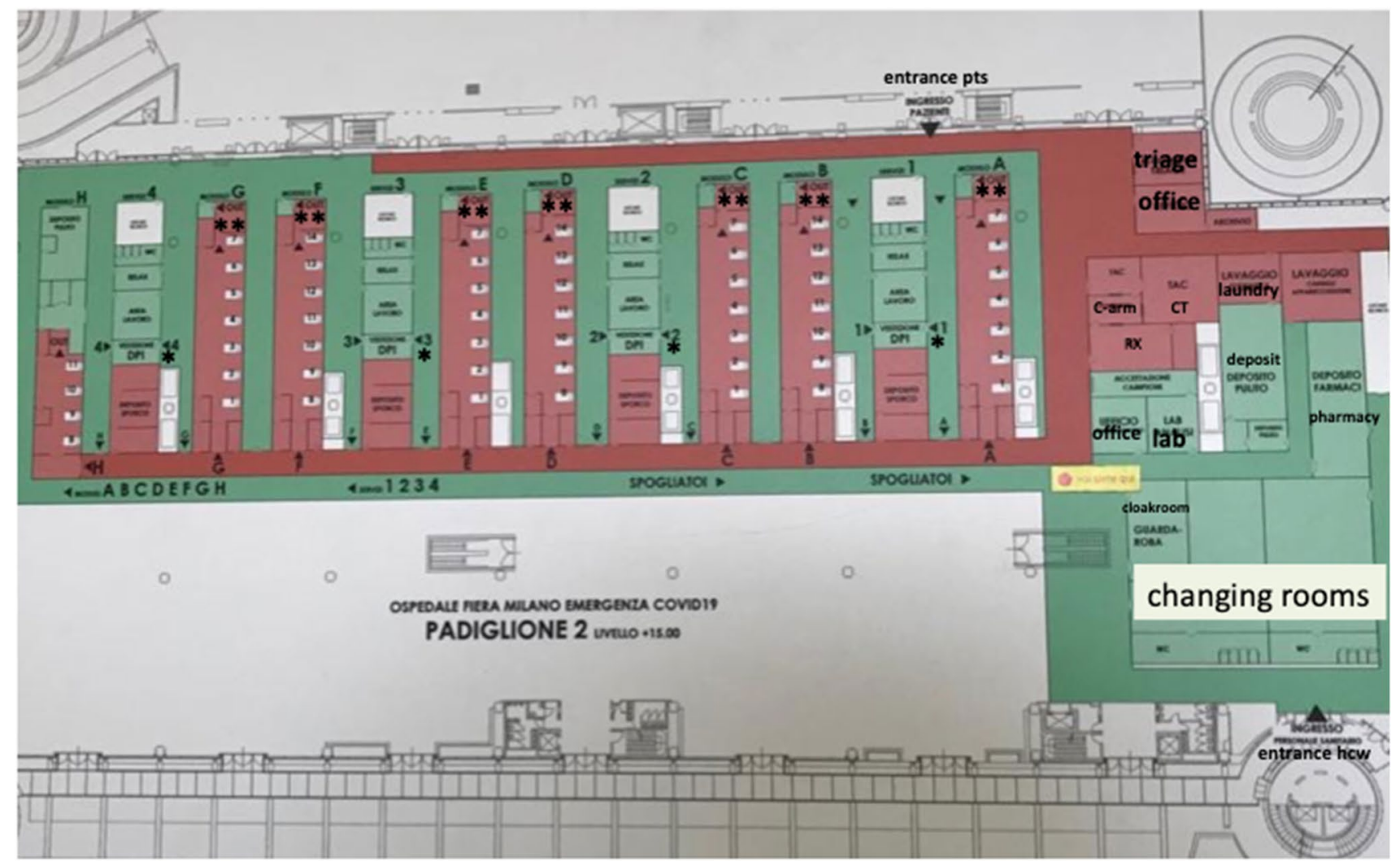

Fig. 1 Map of one of the two floors: red and green zones are evident. lab laboratory, hcw healthcare workers, * donning PPE point, ** doffing PPE point

and easy to decontaminate and serves as the mainstay imaging tool for emergency departments and inpatient settings. Therefore, portable radiography equipment is present in predefined locations. A leaded glass barrier (similar to grocery store lines) may serve as an extra barrier for staff, especially in the field acute care or screening settings. Two CT machines are present, one for each floor: they are located in the red zone and closer to the triage to permit immediate access to the CT room, even for patients just admitted. A little room with a workstation is located closer to the CT room, but staffs are encouraged to do remote communications, remote interpretations, and remote consults whenever possible. In the green zone, a radiology office is present, in which 2 workstations are available to allow radiologists and residents to interpret images, do reconstructions, make a report, and be accessible via remote communications to the red zone staff. A workstation dedicated to artificial intelligence (AI) is also available to permit studies on chest X-rays and CT scans, for research purposes and point of care applications of novel approaches to multi-parametric data integration, all with regulatory clearances and a network of multinational partnerships.

\section{PPE}

As directed by Government policies, guidelines, and procedures, surgical masks must be worn by anyone who leaves home. Overall, shoe covers, clogs, and surgical masks are mandatory throughout operations, even in the green zone. Before entry into the red zone, special PPE must be worn. Screening may occur upon entry to the hospital, entry into the green zone, or entry into the red zone.

All healthcare workers involved should have extensive knowledge and training in the correct donning and doffing steps, along with appropriate and safe disposal of PPE. This requires training and practice sessions for these critical steps and procedures. Videos and guidance during the donning and doffing were dedicated for every operator, and each operator was trained by senior mentor, until he/she became fully independent with verifiable metrics and "certification" by a mentor. Written protocols should be available and posted in every donning and removing room and on hand for all operators and staff. Careful hand hygiene, correct wearing of protective equipment, and strict adherence to infection control procedures ensured continued 


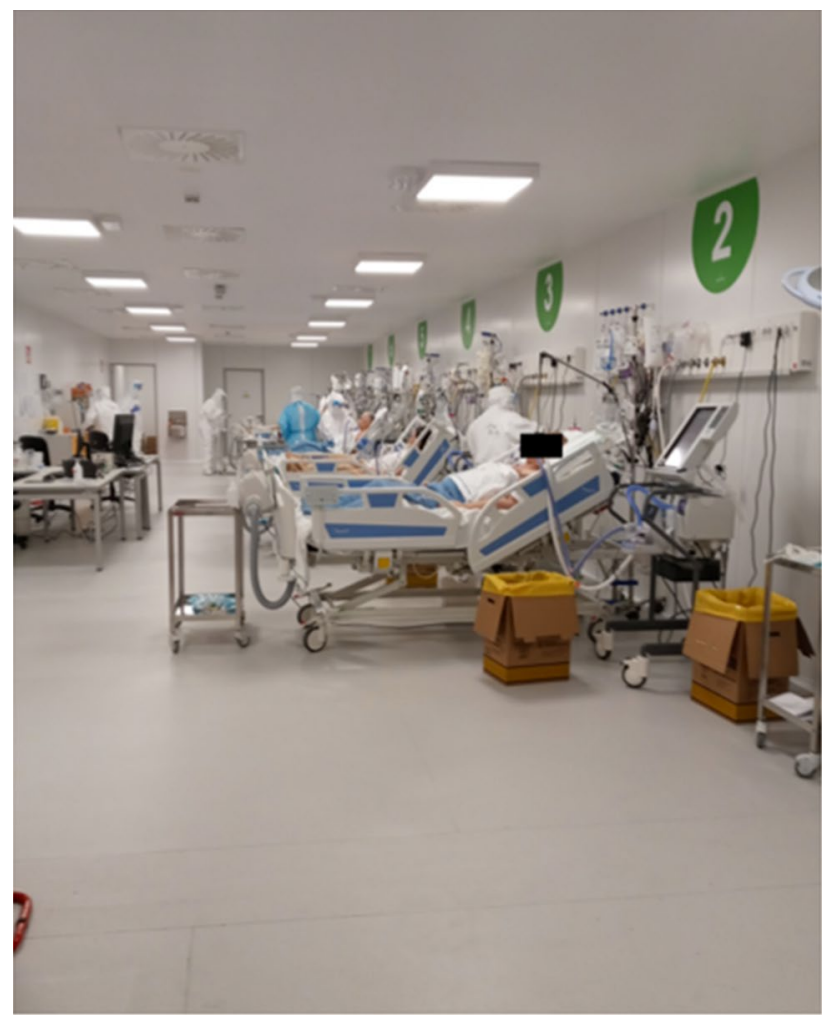

Fig. 2 Example of beds in each module

and complete compliance. Non-powdered latex-free gloves should be used by all operators. Eye protection and face shielding should be used. Gowns should be long-sleeved and made of non-absorbable (fluid-resistant) materials. The same gown should not be worn for all patients. In case gowns are not available, waterproof aprons should be used.

The WHO recommends the use of particulate respirators (N95 or FFP2 or equivalent) for contact and airborne precautions [4]. PPE shortages should be met timely with a coordinated approach and plan endorsed by hospital epidemiology experts to minimize risk to staff and patients. Although beyond the scope of this report, this may be the most critical piece of the puzzle in some pandemic settings. Close communication with epidemiology is encouraged.

\section{Workshift}

Shifts are employed to provide around the clock coverage for radiologists, interventional radiologists, and technologists, with as many services as possible performed remotely, via teleradiology technology. Workloads should be matched whenever possible to the workforce, realizing this may be impossible during a pandemic setting. Efforts to limit around the clock work should be made to promote eating, sleeping, and daily activities to allow for minimization of fatigue induced errors or breaches in techniques, for example.

Whenever possible, teams of physicians, nurses, and technologists should work together, or as staffing allows, in staggered shifts as teams, in order to minimize potential transmission between teams, or from one patient to many staff all at once. Careful attention to avoid group mealtimes or coffee times is made using social distancing guidelines, since mask removal is often required for such activities, which creates vulnerabilities for transmission. Drinking straws are encouraged, and $2 \mathrm{~m}$ distancing is encouraged whenever possible, regardless of mask use. Staff who enter new spaces are encountered with screening queries upon entry, to ensure the critical in-person requirement for their entry.

\section{X-ray}

The hospital is dedicated to bedridden patients; therefore, only portable X-ray machines are present. Fourteen portable radiography systems are available for each floor. Red zone portable equipment remains in the red zone; they are never brought out. Technicians are usually working in their office in the green zone, and they may be called by a phone call directly by the red zone anaesthesiologists or critical care staff. Anaesthesiologists designate the necessary X-ray studies and indicate the number and the exact locations of the patients (module, beds number, etc). Radiology technologists (usually 2) may access the red zones through the changing room, where they can donn PPE, via a PPE Donning SOP.

A station to process images and put them online in the PACS is present in the red zone. Therefore, after taking the $\mathrm{x}$-ray, within a few minutes, images are online and all specialists, including radiologists, may visualize them. Radiologists are usually in the green zone and may produce a report from the workstation.

\section{US}

In each module, a dedicated US machine is present (Fig. 3), each fully equipped with 3 probes. US machines may be used by anaesthesiologists for vessel image-guided puncture for central vein accesses, for catheter placement, or arterial access for blood gas analysis. Cardiologists and/or anaesthesiologists may use the probe dedicated to cardiac ultrasonography or echocardiography. A convex probe is available for thorax or abdomen fast US examinations usually performed by non-radiological specialists, or by the interventional radiology team for assessment for bedside procedures if necessary. The radiologist may be called to perform a dedicated US if a specific diagnostic question 


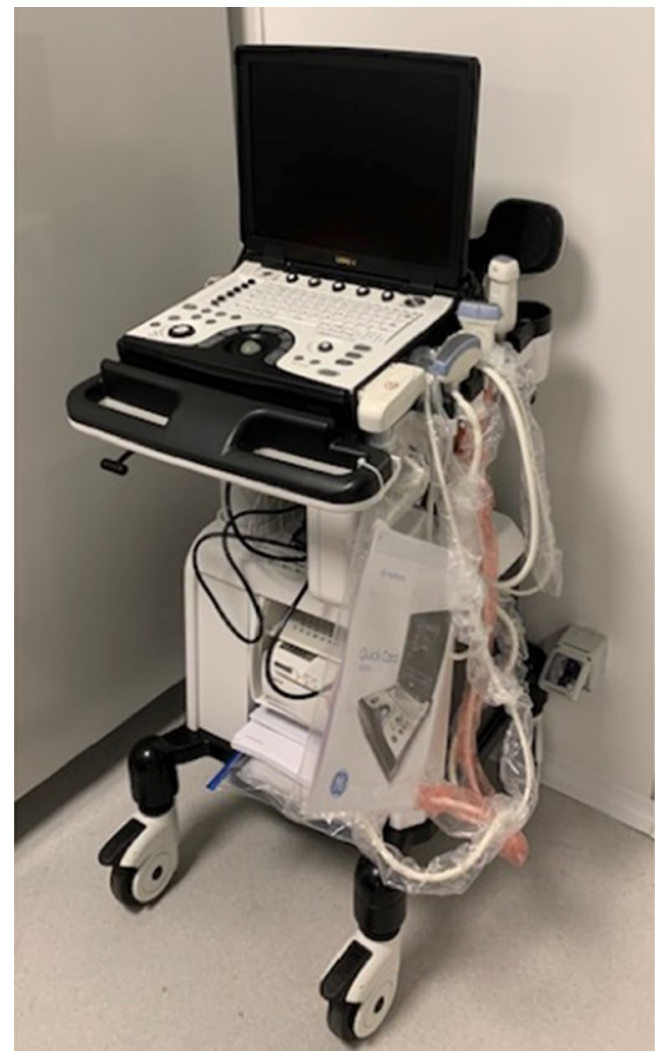

Fig. 3 US machine

arises. Recently, lung ultrasound has rapidly become a tool for monitoring of patients stricken by the novel COVID-19, helping in clinical decision making and reducing the use of both chest x-rays and computed tomography (CT) [5]. US may provide a rapid, continuous, and real-time assessment of peripheral lung physiology, such as fluid in peripheral infiltrates, interlobular septal thickening, or any adjacent effusions, which may change dynamically.

\section{CT}

CT scanners are located in red zones, as described above (Fig. 4).

The CT room is equipped with a ventilator, intercoms, and cameras for dedicated monitoring and patient and staff communications during CT execution. The pump injector and any other room surfaces are completely covered during exams. At any time, multidisciplinary decision making and team assessments may occur, preferably remotely, with anaesthesiologist, critical care staff, and diagnostic and/or interventional radiologists to discuss clinical indications or findings. Decision making is always a team effort.

Once the decision is made that a CT scan is clinically indicated, after donning PPE, a radiologist and

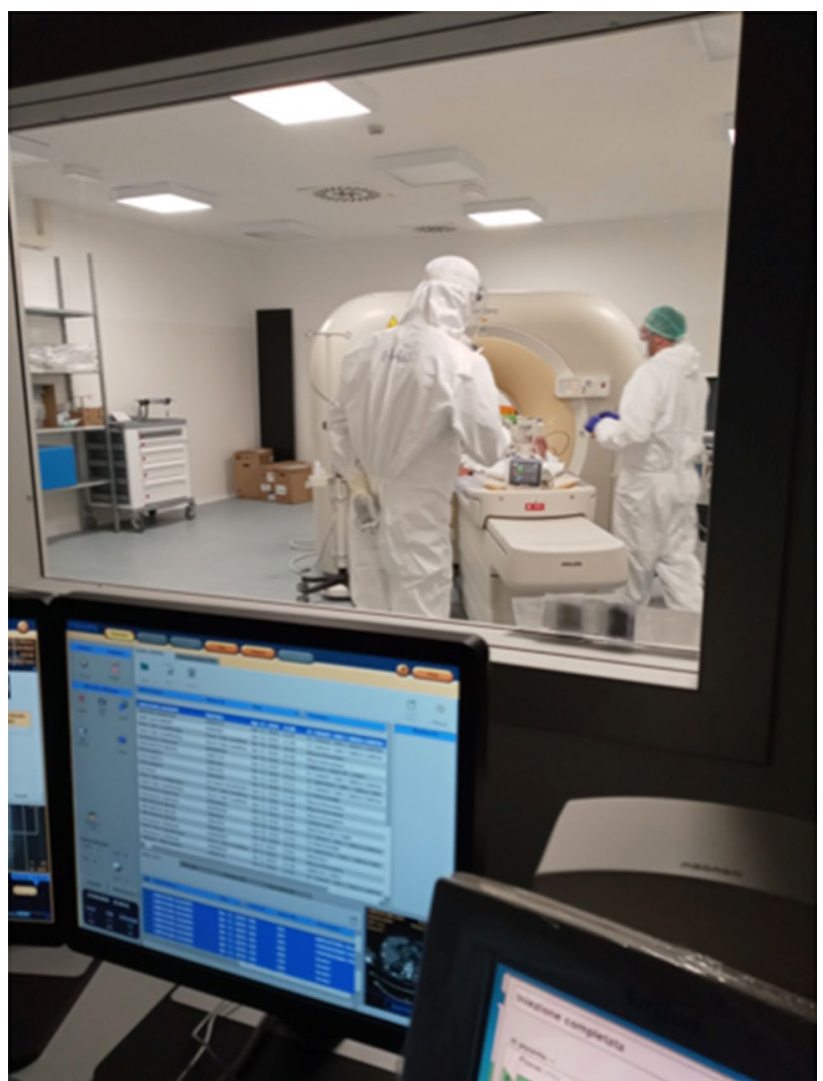

Fig. 4 CT scan

technologist may enter the red zone. Radiologists have the opportunity to decide and protocol the exam. Images are immediately available on hospital PACS for all the specialists in charge. Reports may be done directly in the red zone or when the radiologist will be back in the green zone office. Exchange between zones is governed by strict adherence to SOPs.

A commercial disposable portable isolation bag was designed for outpatients by radiologists (National Institutes of Health with Cvico Medical Solutions (USA) (Fig. 5a, b). The isolation bag use in any patient who is not intubated will allow for oxygen exchange and minimization of viral load on contaminated surfaces. Air exchange rates (and negative flow ideally) should be closely analyzed prior to implementation of such facilities, to ensure that adequate passive air exchange is allowed in between patients, especially important in the event that COVID-19 status is uncertain, or for standard practices outside of the red zone, or in outpatient or acute care facilities. In this specialized hospital built for isolation patients, this device may not be necessary, but in future projects and managing non-intubated patients, this may permit a CT area to be cleaner when "not isolated." 
Fig. 5 a, b Disposable CT isolation bag to reduce room and equipment contamination in diagnostic or interventional scenarios. Courtesy Civco Medical Solutions, Kalona, Iowa, USA and National Institutes of Health, Bethesda, MD USA. Reproduced with permission (manuscript under review)
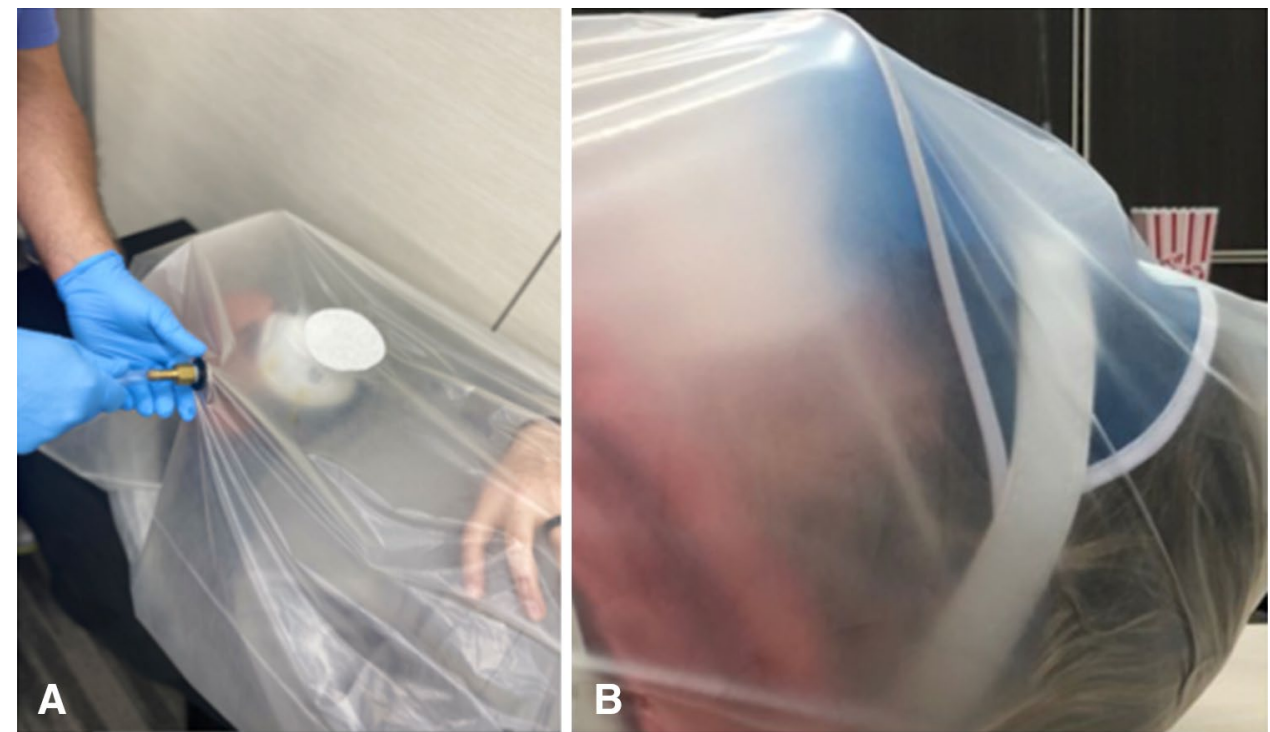

\section{Portable C-arm and interventional radiology}

Closer to the CT room, a suite equipped with a modern portable $\mathrm{C}$ arm is available (Fig. 6). The logistics are strategic, because after execution of CT scan, an interventional radiology procedure may sometimes be immediately indicated. Such proximity minimized patient transfers and staff exposure risk.

An important concept must be kept in mind: all the devices in the red zone should never leave it nor be brought in the green zone. Therefore, interventional radiologists, together with technologists, prepared small dedicated kits for each procedure. In the green area, a little collection of spare materials is provided (catheters, microcoils, drainages, guidewires, etc).

Interventional radiologists may access to the room equipped with portable $\mathrm{C}$-arm only after donning PPE, since located in the red zone.

The kits available in red zone include materials and devices for embolization, inferior vena cava filter deployment, pharmacological and mechanical fibrinolysis, percutaneous drainage deployment, thoracentesis, paracentesis, chest tube placement, and central vein access deployment.

For hybrid procedures, US machines may be taken from one of the modules, but must undergo strict decontamination SOP in between patients, despite the red zone location.

Fig. 6 Portable C-arm

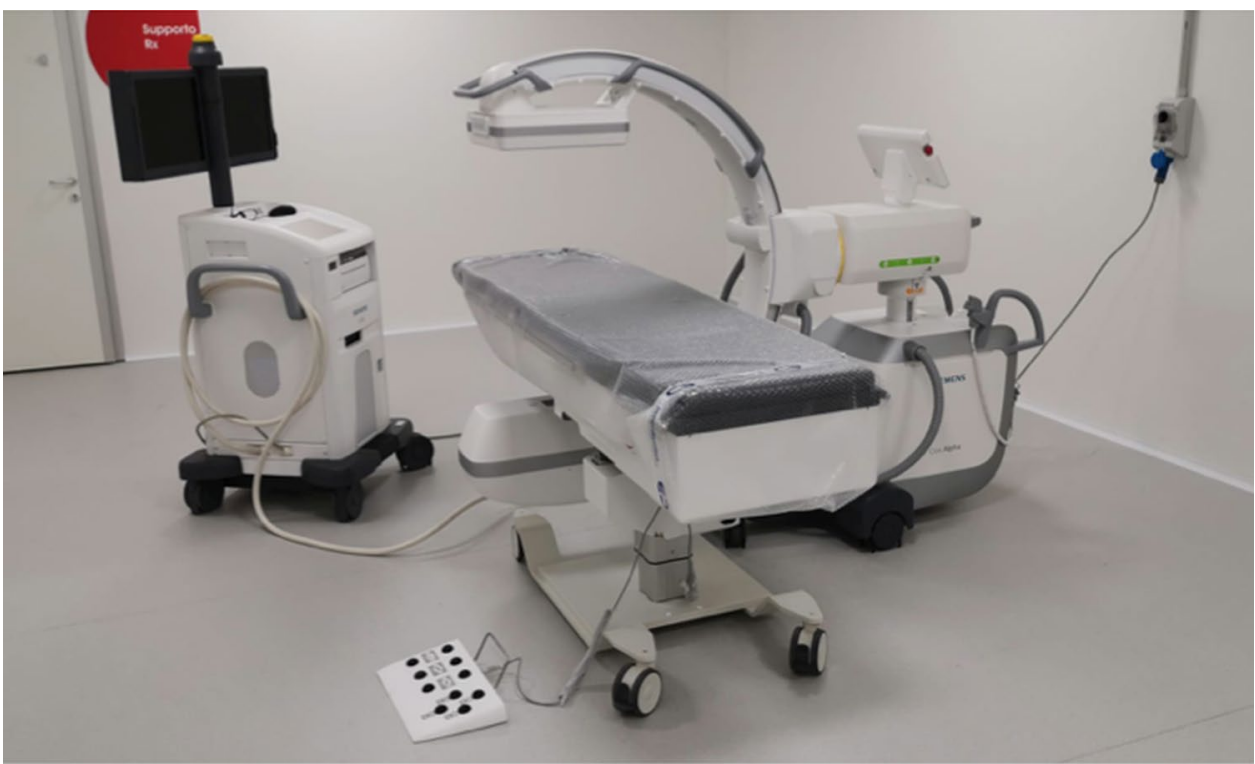




\section{Cost impact of investment}

Such a dedicated facility is a costly endeavor indeed. However, such as effort should be carefully analyzed with cost-effectiveness and cost-utility metrics that have longterm comparisons and outcomes analyses, based upon the economic realities of the pandemic. The cost metrics should factor in the downstream cost of lives saved, quality adjusted life years saved, pain averted, and intact family units toward the strength of the fabric of society at large. Each transmission averted by such a dedicated COVID19-specific facility has the potential to avoid a long-term admission, ventilator use, and resources required for that admission, and each of the contacts for that COVID-19 transmission, with an $R \geq 2$. In a cramped and "mixed" healthcare facility without dedicated team focused on isolation efforts, the staff, patients, and processes might unfortunately mix together. This could allow the virus to spread rapidly, potentially infecting health care workers and patients, and taking health care workers out of the care team. This might occur when that team is already stressed to the max, and short staffed, with demand outpacing supply across multiple fronts of staff, equipment and testing. The infection rate is likely much higher for health care workers, where a single infected worker could also spread the virus to more than 2.2 naive patients. Thus, the downstream costs of a transmission in the healthcare setting are difficult to measure. Thus, each and every averted transmission saves the healthcare system immensely. When confronting a pandemic faceless enemy, focal investments toward a dedicated facility have immense economic benefits that make it overwhelmingly cost effective, especially in the early phases of a pandemic outbreak curve whose end remains blurry and ill-defined. Earlier economic restart possibly made possible by fewer nosocomial transmissions and ideal and early critical care, is also hugely impactful economically. Such investments may be made by proactive and forward-thinking stakeholders and leaders in radiology and policy-making.

\section{Conclusion}

A dedicated facility for the management and triage of patients with COVID-19 clusters the expertise and tools requisite for optimal care. When resources allow for a dedicated ICU facility to be built, this enables team sequestration and collective isolation of staff teams, processes, patients, and equipment. The construction of this COVID19 hospital also allowed relatively easy transfers to and from other ICUs of the Milano area, with strict SOPs.
An environment completely dedicated to isolated COVID-19 patients permit a better management of these patients and resources and promotes containment within the healthcare facility that must care for these COVID-19 patients during a pandemic outbreak. Such prophylactic biocontainment is cost-effective and provides value for the investment in the long term, even if it is a resourceintensive affair in the short term. Repeated practice sessions, training, and standardized and epidemiologically rational SOPs and preparation are paramount. Many hours spent preparing will save lives, as we prepare for this global team battle. Radiology has been viewed as a frontline clinical service. Our presence, knowledge, and expertise are critical. A new critical care outbreak setting for a dedicated radiology and intensive unit for COVID19 was realized. The present experience may be useful for other countries not yet frontline in the actual pandemic or in possible future surges. All of our collective actions may substantially contribute to global health security, since each and every action by a society, government, healthcare ministry, hospital leadership, or each physician and healthcare worker may have profound and downsteam implications and impact for the neighbors and contacts of everyone, for the near future. We are all in this together. United we rise, divided we fall.

Acknowledgements The opinions are those of the authors in their personal capacity and do not necessarily represent the opinions of the National Institutes of Health nor the US Government. Supported in part by the Intramural Research Program of the NIH. Devices discussed may not be cleared for any indication by the CE Mark or the US Food and Drug Administration.

Funding This study was not supported by any funding.

\section{Compliance with Ethical Standards}

Conflict of interest The authors declare that they have no conflict of interest. NIH may have intellectual property related to the isolation bag device.

Ethical approval All procedures performed in studies involving human participants were in accordance with the ethical standards of the institutional and/or national research committee and with the 1964 Declaration of Helsinki and its later amendments or comparable ethical standards.

Consent for publication All authors give their consent for publication.

\section{References}

1. Zou L, Ruan F, Huang M et al (2020) SARS-CoV-2 viral load in upper respiratory specimens of 186 infected patients. N Engl J Med 382:1177-1179

2. http://opendatadpc.maps.arcgis.com/apps/opsdashboard/index .html\#/b0c68bce2cce478eaac82fe38d4138b1 
3. https://www.bancaditalia.it/pubblicazioni/economie-regio nali/2018/2018-0023/1823-eco-regioni.pdf

4. Rational use of personal protective equipment for coronavirus disease 2019 (COVID-19): interim guidance; 27 February 2020, World health Organization.https://apps.who.int/iris/bitst ream/handle/10665/331215/WHO-2019-nCov-IPCPPE_use2020.1-eng.pdf

5. Vetrugno L, Bove T, Orso D, et al (2020) Our Italian Experience Using Lung Ultrasound for Identification, Grading and Serial
Follow-up of Severity of Lung Involvement for Management of Patients with COVID-19 [published online ahead of print, 2020]. Echocardiography https://doi.org/10.1111/echo.14664

Publisher's Note Springer Nature remains neutral with regard to jurisdictional claims in published maps and institutional affiliations. 